\title{
One-Step Synthesis of Colloidal Quantum Dots of Iron Selenide Exhibiting Narrow Range Fluorescence in the Green Region
}

\author{
Pradyumna Mulpur, Tanu Mimani Rattan, and Venkataramaniah Kamisetti \\ Department of Physics, Sri Sathya Sai Institute of Higher Learning, Prasanthinilayam 515 134, India \\ Correspondence should be addressed to Venkataramaniah Kamisetti; kvenkataramaniah@sssihl.edu.in
}

Received 24 August 2013; Revised 7 November 2013; Accepted 8 November 2013

Academic Editor: Mingwang Shao

Copyright ( 2013 Pradyumna Mulpur et al. This is an open access article distributed under the Creative Commons Attribution License, which permits unrestricted use, distribution, and reproduction in any medium, provided the original work is properly cited.

\begin{abstract}
The instantaneous isolation of green fluorescent colloidal quantum dots of iron selenide capped with biocompatible oleic acid is reported in this study. These iron-containing quantum dots also serve as a safe alternative to the conventionally used metalchalcogenide systems in which the heavy metal component is usually toxic. The isolated colored colloidal solutions exhibited intense green fluorescence on exposure to ultraviolet light, which was also confirmed by photoluminescence spectroscopy. The isolated product was subjected to dynamic light scattering and transmission electron microscopy, and the particles were found to exhibit spherical morphology with an average diameter of 6-8 nm, confirming the isolation of quantum dots. The isolated iron selenide quantum dots have promising potential towards bioimaging and sensing, due to the biocompatible coating of oleic acid and iron, which also allows possibility of further chemical derivatization.
\end{abstract}

\section{Introduction}

Quantum dots (QDs) have emerged as one of the most fascinating systems in the new millennium. They have garnered widespread interest amongst a multitude of research groups as they exhibit higher extinction coefficients, higher quantum yields, less photobleaching, broad absorption, and narrow emissions, which can be tuned with size $[1,2]$. Due to these properties they are used in infrared photodetectors, solid state lasers, photovoltaics, QD-LEDs, medical imaging, targeting, therapy, and sensing [3-6]. The other major advantage of QDs is that they can be functionalized with a variety of molecules like antibodies, aptamers, proteins, DNA, and so forth, to enable specificity for early and rapid detection [7, 8]. Moreover in comparison to organic fluorophores which display high photobleaching and low extinction coefficients, QDs are definitely the more suitable alternatives.

The impressive investigations on these systems in almost every discipline are reflected in the work being carried out globally. In addition to the important aspect of discovering novel materials; attempts are constantly being made, to explore new routes of synthesis and functionalization.
Several specific quantum dot systems have been fabricated with an aim towards targeting special applications. Most of the synthesized systems fall under the category of metal chalcogenides, and typically synthesized are the II-VI systems [9]. They include cations of zinc, cadmium, and mercury, combined with anionic oxygen, sulphur, selenium, or tellurium that exhibit a high degree of photoluminescence due to the presence of a direct band gap [6]. CdSe, ZnSe, PbSe, $\mathrm{CdTe}, \mathrm{HgTe}, \mathrm{PbS}$, InP, and $\mathrm{ZnO}$ are some of the extensively studied systems, reported with a high quantum yield [6].

Motivated by the extraordinary benefit of these systems, we made efforts to isolate oleic acid capped iron selenide quantum dots via the hot injection route, which has not been reported previously. Oleic acid has been previously reported to be biocompatible [10]. Iron selenide has interesting optical, electronic, and magnetic properties that could be used for biological imaging or sensing. It shows promising potential as a QD system since iron has a well-defined magnetic domain structure [11]. This would make iron selenide QDs, a probable candidate for targeted delivery under the influence of a magnetic field. It can even be used for applications like MRI for which quantum dot systems are already being 
tested [12]. Moreover, alternative quantum dots systems of $\mathrm{Cd}, \mathrm{Pb}$, and $\mathrm{Hg}$ are known to be toxic [13]. The aim is to isolate stable surfactant capped iron selenide colloidal QDs as a possible biocompatible replacement for the harmful fluorescent markers.

Several methods of synthesis for such quantum dot systems are described in the literature, which are generally chosen so as to have the following properties: monodispersity, chemical integrity and specificity, possibility of further functionalization, and high degree of crystallinity without any defects [14]. Our present work is based on the precursor route, where the principal synthetic strategy of arrested precipitation is used [14]. It is a one pot synthesis route where all the precursors prepared in solvents are added in one vessel at elevated temperatures. This is also known as the hot injection route, which minimizes the number of steps producing the product instantaneously. At some stage of the growth, the surface is stabilized by making use of surfactants or capping agents. Further growth is thereby controlled or arrested as the surfactants selectively bind to the surface of the growing nanocrystal [15]. In our procedure we have suitably modified the precursor route by incorporating a reducing agent, hydrazine monohydrate. The reducing agent is added to prevent the susceptible oxidation of iron which may otherwise prevent the formation of quantum dots. More importantly hydrazine hydrate can also form complexes with the precursors, a critical step in the product formation, as discussed in the subsequent sections.

The final isolated product was characterized by UV, PL, DLS, TEM, and FT-Raman to ascertain the optical properties, size, and chemical composition.

\section{Experimental}

2.1. Chemicals. $\mathrm{FeCl}_{3}, 98 \%$, Otto (Chemika Biochemika Reagents); 1-octadecene, 95\%, trioctyl phosphine (TOP) 90\%, Sigma Aldrich; oleic acid, pure, Merck; hydrazine monohydrate, 99\%, Alfa Aesar; and Selenium powder, HiMedia.

2.2. Synthesis of Iron Selenide Quantum Dots. About $30 \mathrm{mg}$ of selenium powder was added to a beaker containing $5 \mathrm{~mL}$ of 1-octadecene. Subsequently $0.4 \mathrm{~mL}$ of TOP was added to this mixture using a glass syringe. The entire mixture was subjected to mild heating with simultaneous magnetic stirring until a clear solution was obtained. The solvent chosen for this was TOP because it has a high thermal stability and can coordinate with inorganic surfaces easily [16] thereby forming the selenium precursor.

In the modified hot injection route $13 \mathrm{mg}$ of $\mathrm{FeCl}_{3}$ is weighed and added into a three-necked round bottom flask $(250 \mathrm{~mL})$. This is followed by the addition of $0.6 \mathrm{~mL}$ oleic acid, $0.2 \mathrm{~mL}$ hydrazine monohydrate, and $10 \mathrm{~mL}$ octadecene. The round bottom flask is heated to $225^{\circ} \mathrm{C}$ on a heating mantle. The entire working apparatus is set up in a fume hood.

At this temperature, $1 \mathrm{~mL}$ of the prepared Se precursor solution is injected into the round bottom flask. On contact of the Se precursor with the mixture already contained within the flask, an instantaneous colour change is observed. An aliquot of the sample in the flask is immediately drawn and transferred into a storage vial. The drawing of the aliquots is performed repeatedly every 5 seconds until the solution in the round bottom flask is exhausted. Rapid cold quenching of the isolated aliquots after hot injection is performed in crushed ice. This process immediately arrests the reaction, thereby controlling the particle growth and nucleation, preventing further aggregation.

2.3. Characterization. The UV-Vis spectroscopy analysis was performed using the instrument model UV-2450 Shimadzu (Scientific Instruments Corp.), under the photometric absorbance mode. The photoluminescence (PL) analyses were performed using the model LS 55 spectrometer 120 $\mathrm{V}$ from PerkinElmer. The preliminary particle size analyses for the samples were performed with the dynamic light scattering technique (DLS), using the instrument Zetasizer Nano (Malvern Nano ZS) involving the advantageous noninvasive backscatter detection at $173^{\circ}$. The particle size and morphology was determined using TEM, JEOL JEM 2000 FX II. The analysis of the formed product was carried out using FT-Raman spectroscopy (Thermo Nicolet 6700).

\section{Results and Discussion}

The colorless mixture on addition of the Se precursor at $225^{\circ} \mathrm{C}$ immediately transformed to pale yellow. A total of 12 aliquots (with a 5-second interval between drawing the successive aliquots) were drawn from the mother solution in the flask. The samples ranged visibly from a pale yellow color of the first aliquot to a dark brown of the last aliquot as shown in Figure 1(a). The isolated aliquots when placed under UV light exhibited a distinct uniform green fluorescence (Figure 1(b)). This was the first indication of an iron based product displaying narrow range fluorescence in the green region. The entire experiment when repeated several times produced concordant results and the synthesized samples were found to be very stable at room temperature.

In UV-Vis spectrometry, the 1st aliquot displayed maximum absorbance at around $315 \mathrm{~nm}$, the 6 th at $325 \mathrm{~nm}$, and the 12th at around $330 \mathrm{~nm}$ as shown in Figure 2(a). Since the absorption peaks were not separated by a significant shift, it led us to ascertain that the synthesis yielded products exhibiting a fairly monodisperse size range.

The photoluminescence study of the above aliquots was performed using hexane as the solvent. The three aliquots were subject to excitation at $300 \mathrm{~nm}$ and the emission spectrum was recorded. The 1st and 6 th aliquots displayed maximum emission exhibiting two prominent peaks at around $510 \mathrm{~nm}$ and $530 \mathrm{~nm}$ corresponding to a bluish green and a bright green fluorescence, respectively. The intensity of the $510 \mathrm{~nm}$ peak was higher than that of the $530 \mathrm{~nm}$ peak. The 6th aliquot displayed a very minute red shift in peak position and less difference between the two peak intensities. The 12th aliquot however displayed a single prominent peak, which was broader encompassing the range between $500 \mathrm{~nm}$ to $550 \mathrm{~nm}$; centered at $515 \mathrm{~nm}$, corresponding to green color of fluorescence (Figure 2(b)). 


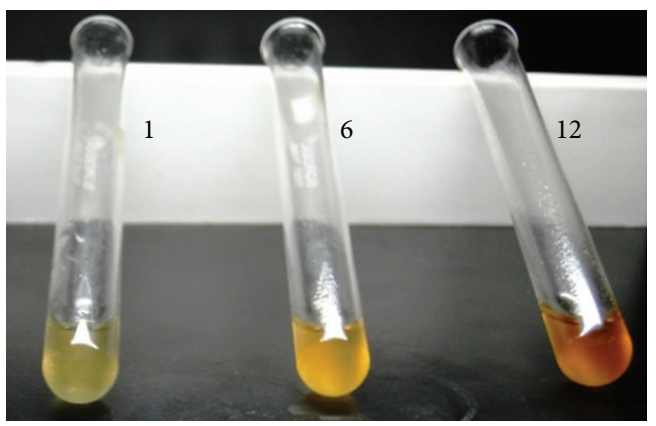

(a)

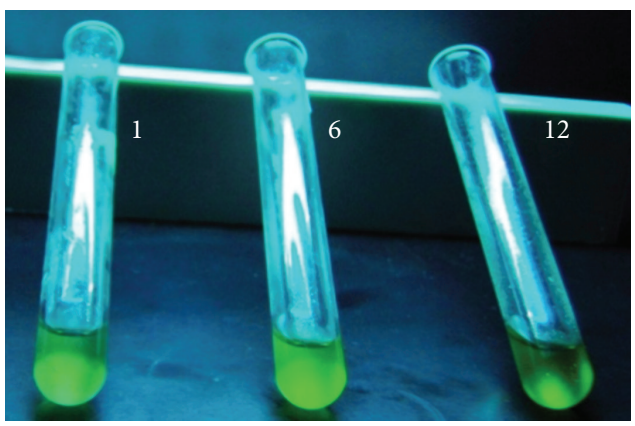

(b)

FIGURE 1: Photograph of the isolated colloidal aliquots of iron selenide under (a) white light and (b) UV light.

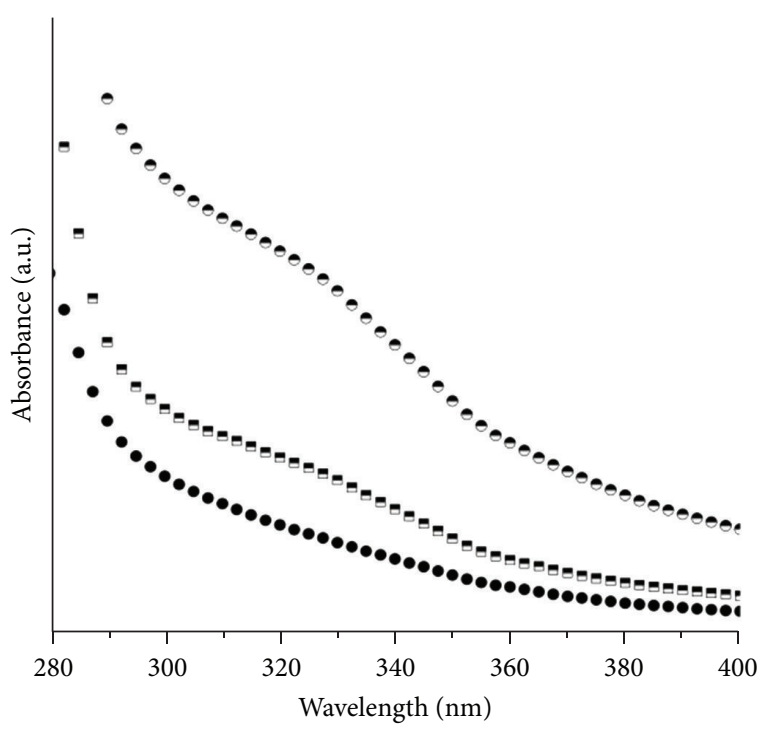

- Aliquot 1

- Aliquot 6

- Aliquot 12

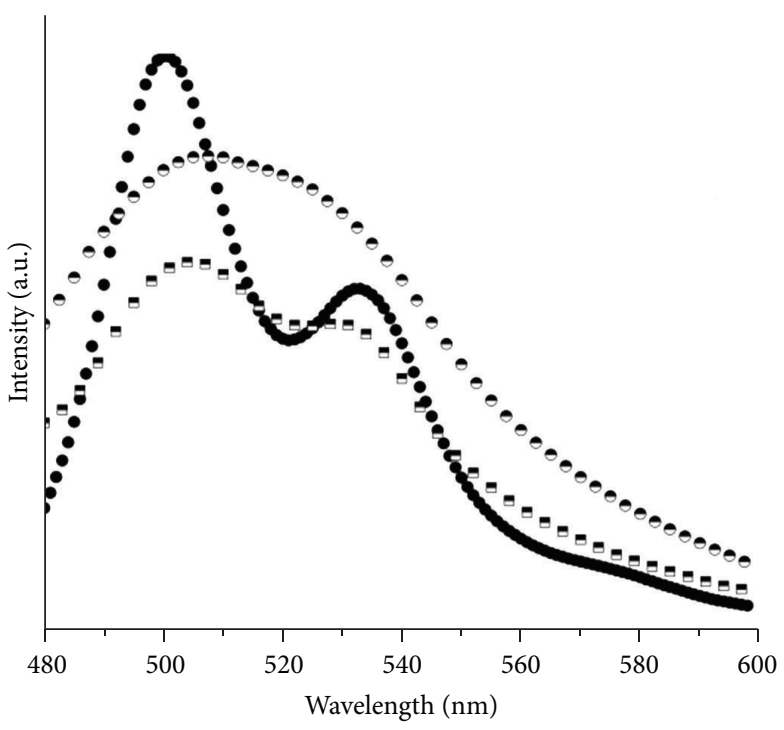

- Aliquot 1

- Aliquot 6

- Aliquot 12

(a)

(b)

FIGURE 2: (a) UV-Vis absorption spectra of isolated colloidal aliquots 1, 6, and 12; (b) photoluminescence spectra of isolated colloidal aliquots 1,6 , and 12 , at $\lambda_{\text {ex. }}=300 \mathrm{~nm}$.

The profile of the peaks can be attributed to the dynamics of the reaction of synthesis, that is, hot injection route. The presence of two prominent peaks for the first aliquot indicates the presence of the product of two different sizes due to the rapid dynamics at the inception of the reaction. The reduced difference in intensity between the two peaks in the 6th aliquot is indicative of a reaction undergoing stabilization. Due to the presence of the surfactant oleic acid, the newly formed nuclei are capped immediately leading to the growth of the clusters to an optimal size and the reaction stabilizes. This is clearly illustrated in the emission peak of the final aliquot, which is in the form of a broad profile and indicative of a stabilized reaction. The nanocrystals synthesized via this route undergo Ostwald ripening resulting in the production of monodisperse particles in the quantum dot regime which can be ascertained from the well-defined
PL peaks $[15,17]$. Moreover, since the shift between the peaks was minimal from the first to the last aliquot with all the isolated aliquots displaying similar colors of fluorescence, it was confirmed that the synthesized systems exhibit "narrow range fluorescence."

The size of the particles in the colloidal system was ascertained by dynamic light scattering method. The particle size distribution by number data as shown in Figure 3 displays an average size distribution of about $5 \mathrm{~nm}$. This substantiates the formation of stable quantum dots of the iron selenide compound.

To acquire a more resolved image displaying the size and morphology of the iron selenide particles we performed a TEM analysis on the sample. The TEM images clearly display quantum dots, as dark spots enveloped in a surfactant matrix, possessing spherical morphology and diameter ranging from 


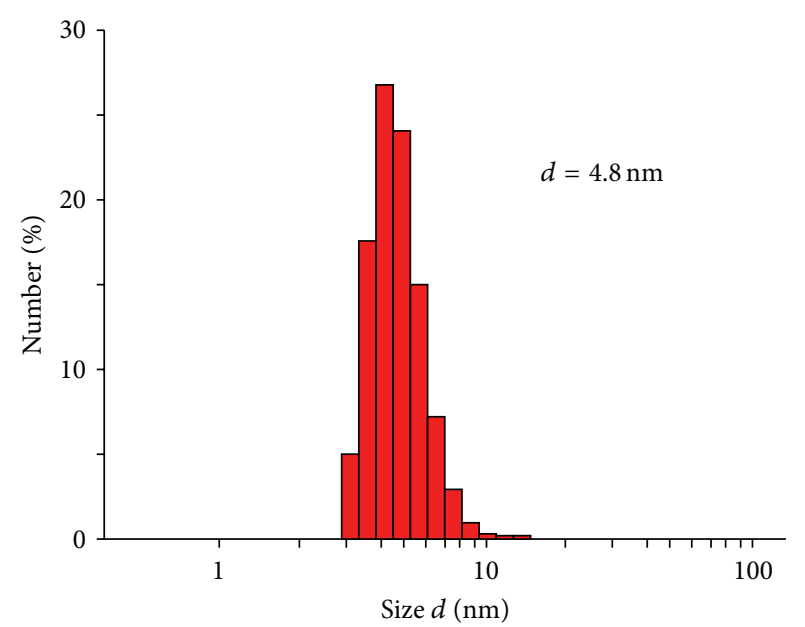

FIGURE 3: DLS data displaying the hydrodynamic radius of iron selenide.

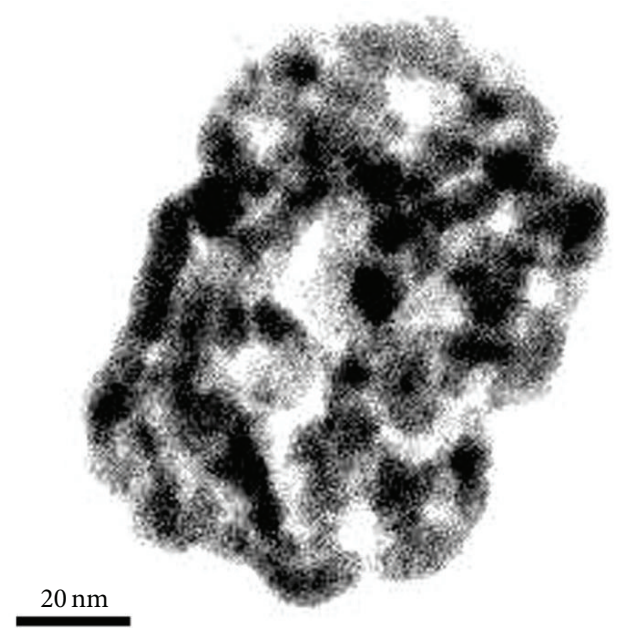

Figure 4: TEM image displaying iron selenide quantum dots with an average diameter of $6-8 \mathrm{~nm}$.

about 5 to $15 \mathrm{~nm}$, with a mean diameter of about $6-8 \mathrm{~nm}$, displayed in Figure 4, which is also in close accordance with the DLS data.

The chemical nature of the isolated quantum dots was established using FT-Raman spectroscopy. The spectra of the solvents display peaks from about $3500 \mathrm{~cm}^{-1}$ to about $1000 \mathrm{~cm}^{-1}$, while the sample spectra displayed an additional distinct band centered at the $220 \mathrm{~cm}^{-1}$ as observed in Figure 5. The line at around $220 \mathrm{~cm}^{-1}$ can be attributed to FeSe bond vibrations corresponding to the $\beta$-FeSe phase of the fluorescent iron selenide QDs [18].

The formation of FeSe can be probably explained by the reaction mechanism involving hydrazine hydrate. In the modified hot injection route, we use hydrazine monohydrate, which as a reducing agent effectively reduces $\mathrm{Fe}^{3+}$ from the iron salt to $\mathrm{Fe}^{2+}$ and the chalcogen to chalcogenide anions, that is, Se to $\mathrm{Se}^{2-}$ [19]. In aqueous solution hydrazine exists

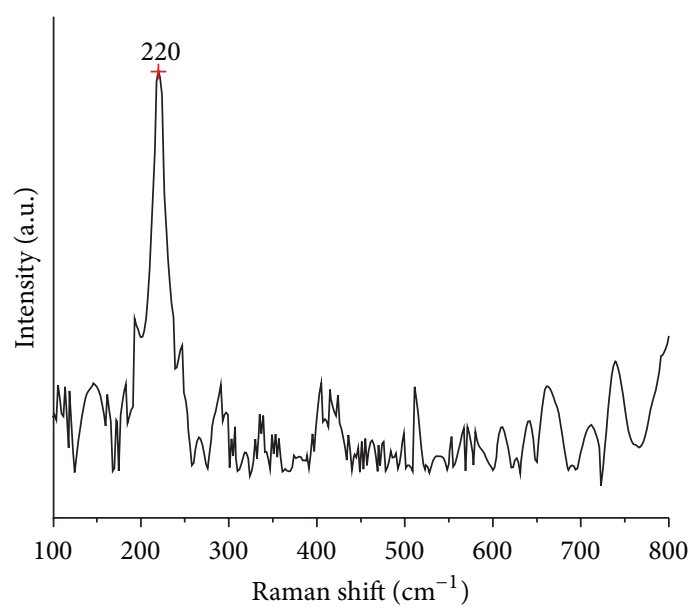

FIGURE 5: FT-Raman spectra of iron selenide quantum dots displaying the characteristic $220 \mathrm{~cm}^{-1}$ band corresponding to the $\beta$-FeSe phase.

as hydrazinium and hydroxyl ions, as shown in (1). In this experiment the concentration of hydrazine monohydrate is optimized to first reduce the iron salt enabling the formation of $\mathrm{Fe}^{2+}$ :

$$
\begin{gathered}
\mathrm{N}_{2} \mathrm{H}_{4} \cdot \mathrm{H}_{2} \mathrm{O} \longrightarrow \mathrm{N}_{2} \mathrm{H}_{5}^{+}+\mathrm{OH}^{-} \\
\mathrm{N}_{2} \mathrm{H}_{5}^{+}+\mathrm{Fe}^{3+} \longrightarrow \mathrm{Fe}^{2+}+\mathrm{NH}_{4}^{+}+\mathrm{H}^{+}+\frac{1}{2} \mathrm{~N}_{2} .
\end{gathered}
$$

The acidic conditions generated in the reaction vessel further initiate the reduction of selenium:

$$
\mathrm{Se}+2 \mathrm{~N}_{2} \mathrm{H}_{5}^{+} \longrightarrow \mathrm{Se}^{2-}+\mathrm{N}_{2}+2 \mathrm{NH}_{4}^{+}+2 \mathrm{H}^{+}
$$

In this manner, the reaction conditions in the hot injection route, aided by the usage of hydrazine monohydrate as the reducing agent and oleic acid as a capping agent, led to the formation of stable colloidal FeSe quantum dots which are biocompatible.

\section{Conclusions}

Quantum dots have myriad applications chief among them being their role in the delivery of therapeutic agents and imaging of cells and tissues. Thus far, the conventionally used metal-chalcogenide systems are reported to be toxic due to the presence of heavy metals $(\mathrm{Cd}, \mathrm{Hg}$, and $\mathrm{Pb})$ which leach out of the QD system on UV exposure. These led to systemic toxicity in the healthy cells and tissues. In our study, we have been able to isolate green fluorescent iron selenide QDs in a single step. The isolated FeSe quantum dots display strong green fluorescence and have been advantageously rendered biocompatible due to oleic acid capping in addition to the nontoxic nature of iron in biological systems. Consequently these QDs have promising applications towards in vitro or in vivo imaging and sensing protocols, in biosystems. 


\section{Conflict of Interests}

The authors declare that there is no conflict of interests regarding the publication of this paper.

\section{Acknowledgments}

The authors are grateful to Bhagawan Sri Sathya Sai Baba, Founder Chancellor, Sri Sathya Sai Institute of Higher Learning, for being a source of constant motivation and inspiration in all their activities. The authors also thank the support from the Department of Science \& Technology (DST Inspire Fellowship Program), Ministry of Science and Technology, Government of India.

\section{References}

[1] L. Spanhel, M. Haase, H. Weller, and A. Henglein, "Photochemistry of colloidal semiconductors. 20. Surface modification and stability of strong luminescing CdS particles," Journal of the American Chemical Society, vol. 109, no. 19, pp. 5649-5655, 1987.

[2] A. P. Alivisatos, "Semiconductor clusters, nanocrystals, and quantum dots," Science, vol. 271, no. 5251, pp. 933-937, 1996.

[3] M. Grundmann, "Present status of quantum dot lasers," Physica E, vol. 5, no. 3, pp. 167-184, 1999.

[4] T. J. Bukowski and J. H. Simmons, "Quantum dot research: current state and future prospects," Critical Reviews in Solid State and Materials Sciences, vol. 27, no. 3-4, pp. 119-142, 2002.

[5] A. M. Smith, X. Gao, and S. Nie, "Quantum dot nanocrystals for in vivo molecular and cellular imaging," Photochemistry and Photobiology, vol. 80, no. 3, pp. 377-385, 2004.

[6] B. Debasis, L. Qian, T.-K. Tseng, and P. H. Holloway, "Quantum dots and their multimodal applications: a review," Materials, vol. 3, pp. 2260-2345, 2010.

[7] S. Dwarakanath, J. G. Bruno, A. Shastry et al., "Quantum dot-antibody and aptamer conjugates shift fluorescence upon binding bacteria," Biochemical and Biophysical Research Communications, vol. 325, no. 3, pp. 739-743, 2004.

[8] V. Bagalkot, L. Zhang, E. Levy-Nissenbaum et al., "Quantum dot-aptamer conjugates for synchronous cancer imaging, therapy, and sensing of drug delivery based on Bi-fluorescence resonance energy transfer," Nano Letters, vol. 7, no. 10, pp. 30653070, 2007.

[9] J. G. Brennan, T. Siegrist, P. J. Carroll et al., "Bulk and nanostructure group II-VI compounds from molecular organometallic precursors," Chemistry of Materials, vol. 2, no. 4, pp. 403-409, 1990.

[10] J. Zhang, X. G. Chen, L. Huang, J. T. Han, and X. F. Zhang, "Selfassembled polymeric nanoparticles based on oleic acid-grafted chitosan oligosaccharide: biocompatibility, protein adsorption and cellular uptake," Journal of Materials Science, pp. 1-9, 2012.

[11] M. Akhtar, J. Akhtar, M. A. Malik, F. Tuna, M. Helliwell, and P. O'Brien, "Deposition of iron selenide nanocrystals and thin films from tris(N,N-diethyl- $\mathrm{N}$ [prime or minute]naphthoylselenoureato)iron(iii)," Journal of Materials Chemistry, vol. 22, pp. 14970-14975, 2012.

[12] R. Koole, W. J. M. Mulder, M. M. van Schooneveld, G. J. Strijkers, A. Meijerink, and K. Nicolay, "Magnetic quantum dots for multimodal imaging," Wiley Interdisciplinary Reviewsy, vol. 1, no. 5, pp. 475-491, 2009.
[13] A. Hoshino, K. Fujioka, T. Oku et al., "Physicochemical properties and cellular toxicity of nanocrystal quantum dots depend on their surface modification," Nano Letters, vol. 4, no. 11, pp. 2163-2169, 2004.

[14] T. Pradeep, Nano: The Essentials, McGraw-Hill, 2008.

[15] C. De Mello Donegá, P. Liljeroth, and D. Vanmaekelbergh, "Physicochemical evaluation of the hot-injection method, a synthesis route for monodisperse nanocrystals," Small, vol. 1, no. 12, pp. 1152-1162, 2005.

[16] D. V. Talapin, A. L. Rogach, A. Kornowski, M. Haase, and H. Weller, "Highly luminescent monodisperse CdSe and $\mathrm{CdSe} / \mathrm{ZnS}$ nanocrystals synthesized in a hexadecylaminetrioctylphosphine oxide-trioctylphospine mixture," Nano Letters, vol. 1, no. 4, pp. 207-211, 2001.

[17] Ventra, Introduction to Nanoscale Science and Technology, Springer, New York, NY, USA, 2004.

[18] C. E. M. Campos, J. C. De Lima, T. A. Grandi, K. D. Machado, and P. S. Pizani, "Structural studies of iron selenides prepared by mechanical alloying," Solid State Communications, vol. 123, no. 3-4, pp. 179-184, 2002.

[19] Schmidt, Hydrazine and Its Derivatives: Preparation, Properties, Applications, John Wiley \& Sons, New York, NY, USA, 2nd edition, 2001. 

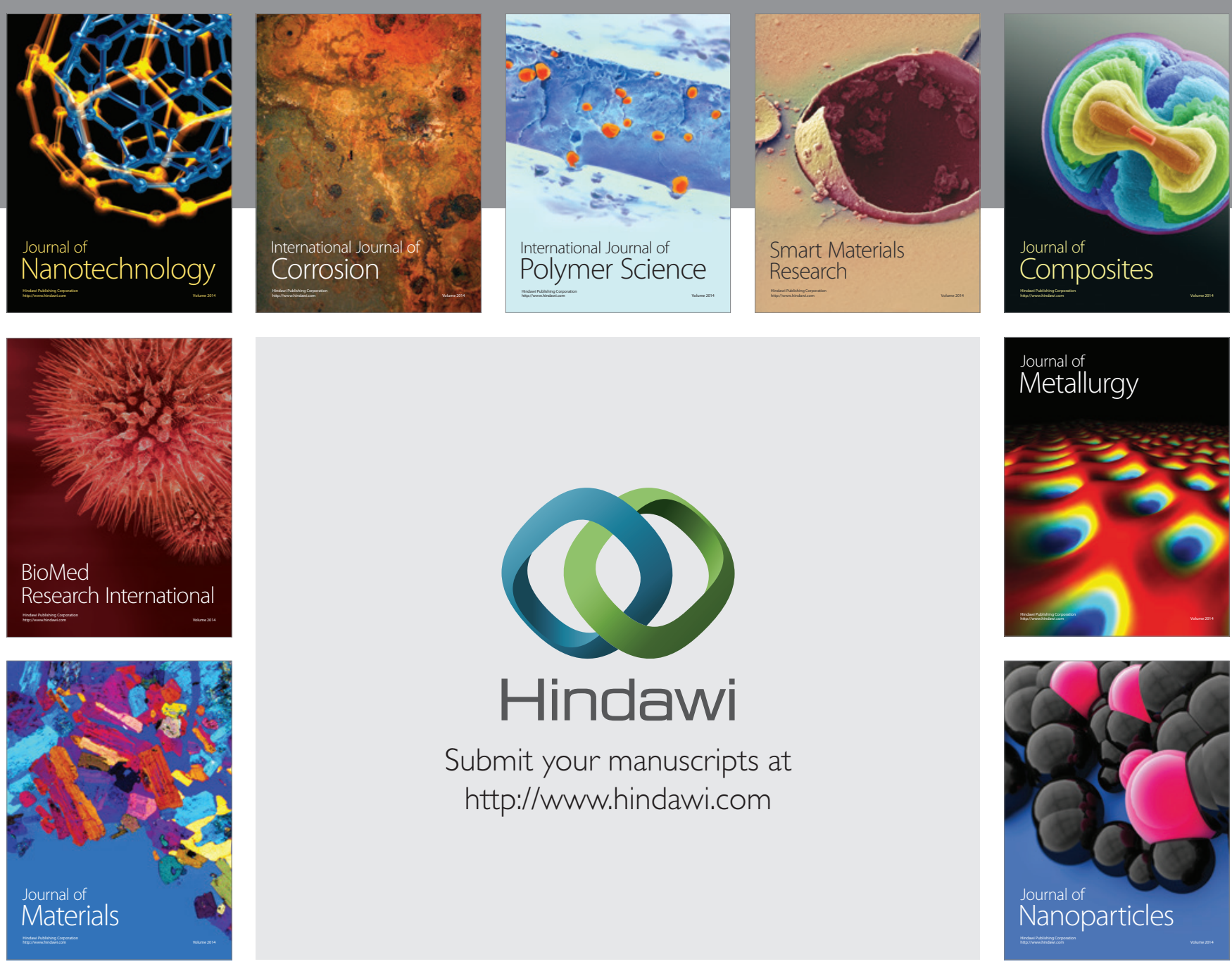

Submit your manuscripts at http://www.hindawi.com
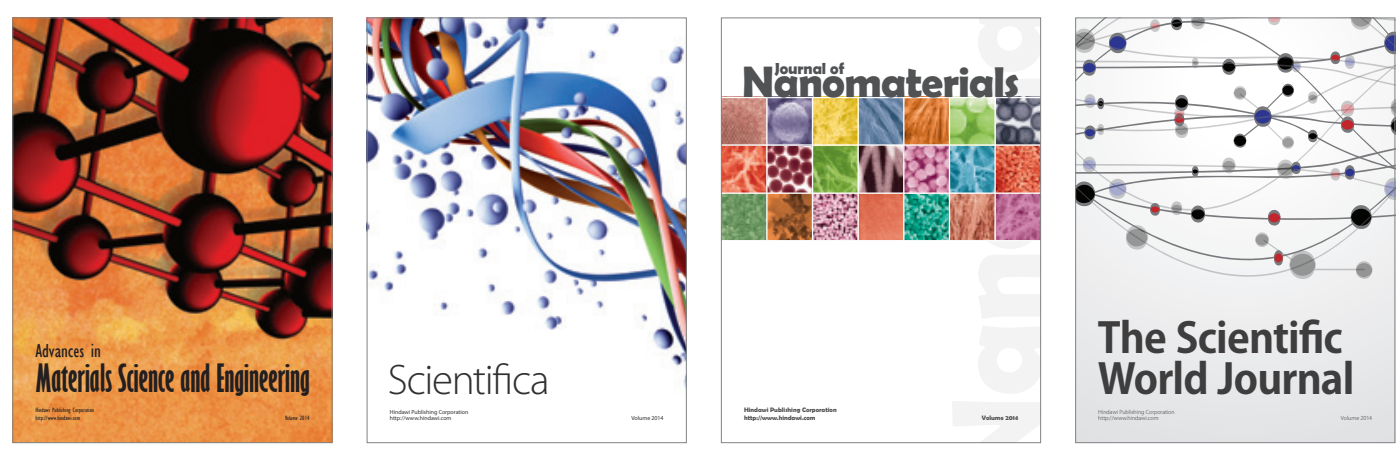

\section{The Scientific World Journal}
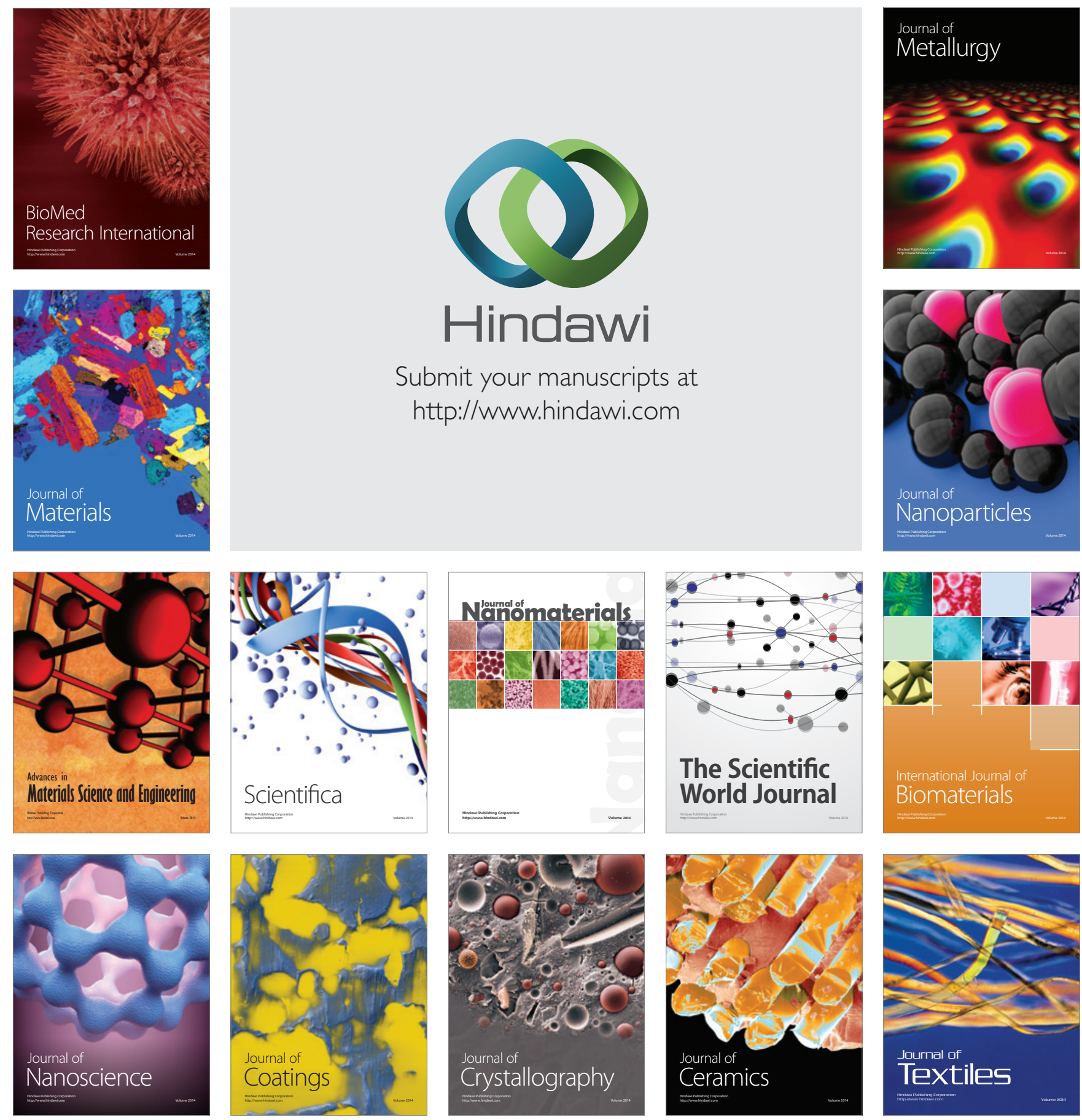